\title{
Optimization of Parametric Periodograms for the Study of Density Fluctuations in a Supersonic Jet
}

\author{
Catalina Elizabeth Stern Forgach, José Manuel Alvarado Reyes
}

Facultad de Ciencias, Universidad Nacional Autónoma de México, México City, México.

Email: catalina@ciencias.unam.mx,jmar@ciencias.unam.mx

Received October $24^{\text {th }}, 2013$; revised November $20^{\text {th }}$, 2013; accepted November $25^{\text {th }}, 2013$

Copyright (C 2013 Catalina Elizabeth Stern Forgach, José Manuel Alvarado Reyes. This is an open access article distributed under the Creative Commons Attribution License, which permits unrestricted use, distribution, and reproduction in any medium, provided the original work is properly cited.

\begin{abstract}
In our research on the density fluctuations of a supersonic jet we were confronted with a quite difficult problem. In the power spectrum obtained either with a spectrum analyzer, the peaks of the two of the modes that we wanted to identify overlapped. We needed to find a signal processing method that would resolve the two main frequencies. We made a thorough investigation of several methods and thought that parametric periodograms were the appropriate tool. The use of parametric periodograms in signal processing requires constant training. The proper application of this tool depends on the determination of the number of parameters that has to be used to best model a real signal. The methods generally used to determine this number are subjective, depending on trial and error and on the experience of the user. Some of these methods rely on the minimization of the estimated variance of the linear prediction error $\sigma_{w p}^{2}$, as a function of the number of parameters n. In many cases, the graph $\sigma_{w p}^{2}$ vs n doesn't have a minimum, and the methods cannot be used. In this paper, we show that there is a strong relationship between $\sigma_{w p}^{2}$ and the frequency resolution $\Delta f$. That is, as we modify $\Delta f$, we obtain graphs of $\sigma_{w p}^{2}$ vs $n$ that present at least one minimum. The spectrum obtained with this optimal number of parameters, always reproduces the frequency information of the original signal. In this paper, we present basically the signal processing of the data obtained in a Rayleigh scattering experiment on a supersonic jet that has also been designed by the authors.
\end{abstract}

Keywords: Parametric Periodograms; Density Fluctuations; Supersonic Jet

\section{Introduction}

Kovasznay in 1953, in a perturbation analysis of Navier Stokes equations, classified weak fluctuations in three independent modes: vortical, acoustic and entropic. Acoustic and entropic modes in a jet are studied by analyzing density fluctuations inside and outside the flow [1-3].

The traditional way to study aero-acoustic noise produced by a jet is through correlations of signals acquired by a three dimensional microphone array in a far-field [4-6], and from there extrapolate to locate the acoustic sources inside the jet.

Besides the extreme complexity of the method, the inverse problem in acoustics does not allow to determine uniquely the source. Also, the diffraction of the acoustic waves by the mixing layer cannot be taken into account.
In the late seventies at the Ecole Polytechnique in France, a non-intrusive optical technique was developed. It can be used as a microphone for a single wave vector [4]. Information about the density fluctuations can be obtained by means of the light scattered by the molecules (Rayleigh scattering) of a transparent gas in motion [4-9].

The signal that is obtained from the photo detector is proportional to the spatial Fourier transform as a function of time of the density fluctuation, for a wave vector given by the optical set-up.

Originally these signals were processed by using a spectrum analyzer. The acoustic and entropic modes should appear as two distinct peaks. The entropic mode corresponds to fluctuations carried by the flow; the acoustic mode should propagate at the speed of sound.

Figure 1 shows the spectrum for fluctuations travel- 


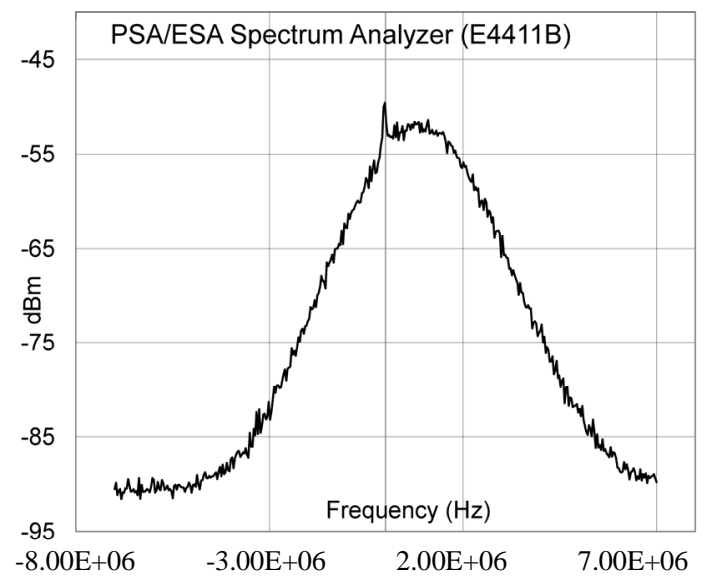

Figure 1. Spectrum obtained by means of the spectrum analyzer. Starting at zero, a lobe describing the density fluctuations within the bandwidth studied may be observed.

ling perpendicular to the flow. In this case, entropic fluctuations should be random about zero, and acoustic wave, for the specific set up, should be at around $2 \mathrm{MHz}$. The spectrum analyzer did not resolve the peaks [5-7].

To ameliorate the resolution, Burg's parametric periodograms were used as a processing tool. Figure 2 shows a comparison between the spectral density obtained by the spectrum analyzer and the one obtained with Burg's periodograms.

The use of the periodograms allowed the identification of both peaks [7-10].

However, the determination of the optimal number of parameters is not an easy task. The methods that exist are based on the number of parameters, which minimize the estimated variance of the prediction error $\sigma_{w p}^{2}$. But there is not always a minimum. We show that it is possible to optimize these methods, when the frequency resolution is considered.

\section{Spectral Density with Periodograms}

Much has been written about periodograms. In 1988, Kay in his book Modern Spectral Estimation, Theory \& Application [12] shows the characteristics of periodograms with examples and programs. In his article Spectrum Analysis, a Modern Perspective [13], he describes a detailed summary of periodograms and explains their properties, advantages and disadvantages. Other authors have also written about periodograms [14-17], highlighting their properties in most of the literature.

John G. Proakis [14] states that "The experimental results given in the references just cited indicate that the model-order selection criteria do not yield definitive results." On the other hand, he states that "It is apparent that in the absence of any prior information regarding the physical process that resulted in the data, one should try different model orders and different criteria and, fi-

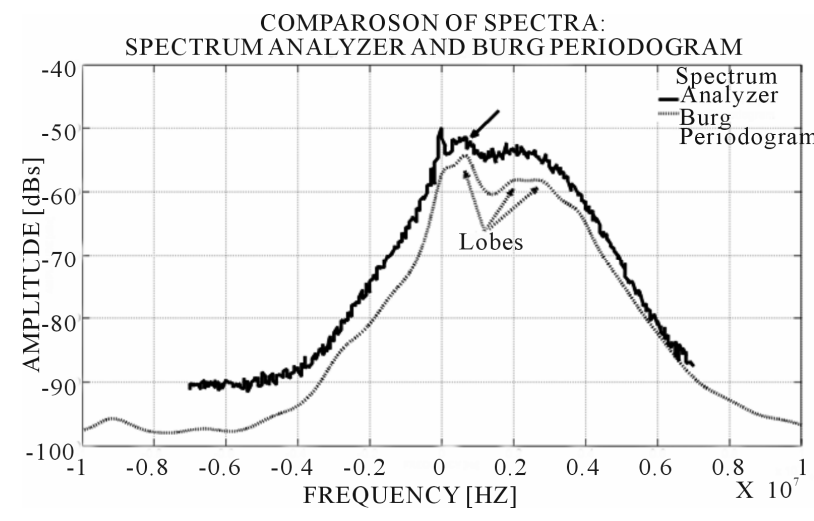

Figure 2. Comparison of spectra. The spectrum analyzer clearly shows one lobe, while the spectrum obtained with the Burg parametric periodogram shows three lobes.

nally, consider the different results". Likewise, Steven M. Kay mentions in his article [13] that "Thus the prediction error power alone is not sufficient to indicate when to terminate the search" and his comment that led to the development of our work is "In the final analysis, more subjective judgment is still required in the selection of order for data from actual processes than that required for controlled simulated computer processes". Proakis and Kay state that, even today, the use of parametric periodograms requires the experience of the user, so the "trial and error" use of these techniques allows a very subjective manner of calculating the number of parameters required to model a given signal. This work proposes that, by considering the frequency resolution, $f_{s} / N$, and using parameter estimation techniques, it is possible to calculate the number of parameters required to model objectively the spectrum of a given signal.

\section{Parameter Selection Techniques}

The ARMA (Auto Regressive Moving Average), AR (Auto Regressive) and MA (Moving Average) models, used by the group of parametric methods to develop algorithms that allow us to estimate the spectral density of a signal are varied, but they all depend on parameter estimation calculations. One of the most important aspects of using the AR model is the selection of the p order. As a general rule, very few parameters flatten the spectrum with few lobes and too many parameters introduce lowlevel spurious peaks in the spectrum [12-16].

In the continued use of periodograms, the practice and "trial and error", is what, until today, makes the user skillful in the application of this type of tools. There are methods that evaluate the "optimal" number of parameters for modeling a signal, based on the determination of the number of parameters that minimize the estimated variance of the prediction error $\sigma_{w p}^{2}$. Table 1 shows some of the most employed methods [11] to determine 
the number of parameters in a parametric periodogram. But, as we mentioned above, even by using the methods of selection of parameters, it is not possible to determine with certainty the appropriate number of parameters to evaluate the spectrum with parametric periodograms.

\section{Applications and Results}

The following exercise will demonstrate how vulnerable are the methods traditionally suggested to determine the number of parameters of a parametric periodogram. For this purpose a signal with four frequency components, 1 $\mathrm{MHz}, 1.01 \mathrm{MHz}, 1.05 \mathrm{MHz}$ and $1.1 \mathrm{MHz}$ is acquired. This signal was sampled at a frequency $f_{s}=5 \mathrm{MHz}$ and has 512 samples. This sampling frequency perfectly meets the Nyquist theorem requirements, in addition of having a frequency resolution $\Delta f \cong 9765 \mathrm{~Hz}$. The number of parameters was evaluated by the methods given in Table 1 and proposed in the literature [11-14]. The results are shown in Figure 3. The linear prediction error depends on the number of samples $N$, and on the number of parameters, $p$.

Both the FPE and AIC methods seem to agree in the minimum in Figure 3; approximately 50 parameters, which minimize $\sigma_{w p}^{2}$. The MDL technique shows a minimum at about 20 parameters.

Figure 4 shows the spectrum of the signal using Burg's parametric periodogram which is compared to the FFT of the time signal. To obtain the Burg periodogram, the number of parameters suggested by the techniques described in Table 1 was used. It should be noticed, that the expected frequency components are not observed. This is the result of the frequency resolution [11,17] obtained with the selected variables, the number of samples and the sampling frequency; they do not allow the differentiation of components standing close by.

To solve the problem, many processing experts could propose to increase the sampling rate or the number of samples. Both criteria are ambiguous and based on experience rather than objectivity.

Our suggestion is to consider the spectral resolution as stated in earlier papers $[11,17]$. We propose to increase the resolution to $\Delta f \cong 305 \mathrm{~Hz}$, and reevaluate the "optimal" number of parameters. Figure 5 shows the corresponding results. From this graph it is possible to distin-

Table 1. Methods for evaluation of the selection of the order $p$.

\begin{tabular}{cc}
\hline FPE: Final & $\operatorname{FPE}(p)=\hat{\sigma}_{w p}^{2}\left(\frac{N+p+1}{N-p-1}\right)$ \\
Prediction Error & $\operatorname{AIC}(p)=\ln \hat{\sigma}_{w p}^{2}+2 p / N$ \\
AIC: A Kaike & \\
Information Criterion & $\operatorname{MDL}(p)=N \ln \hat{\sigma}_{w p}^{2}+p \ln N$ \\
MDL: Minimum \\
Description Length
\end{tabular}

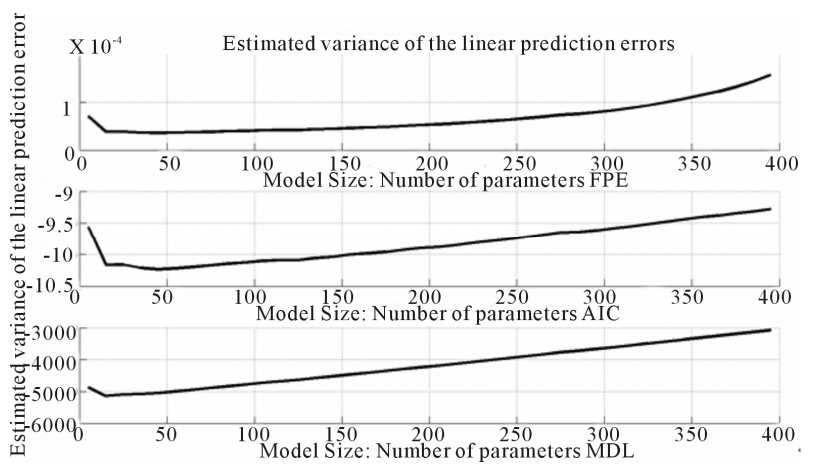

Figure 3. Evaluation of parameter selection techniques, Table 1, for a signal, with four frequency components, $N=512$ and $f_{s}=5 \mathrm{MHz}$.

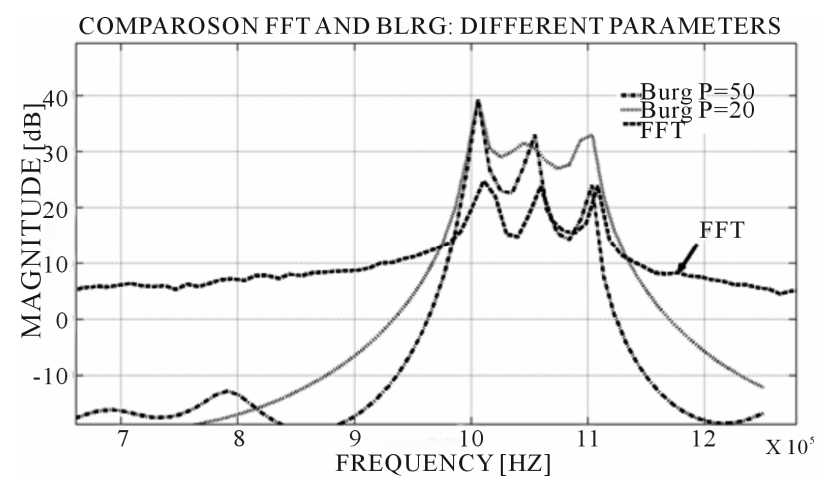

Figure 4. Burg spectra using the number of parameters suggested by parameter evaluation methods, using Table 1 we calculate the number of parameters, and compared against the spectrum obtained by FFT.

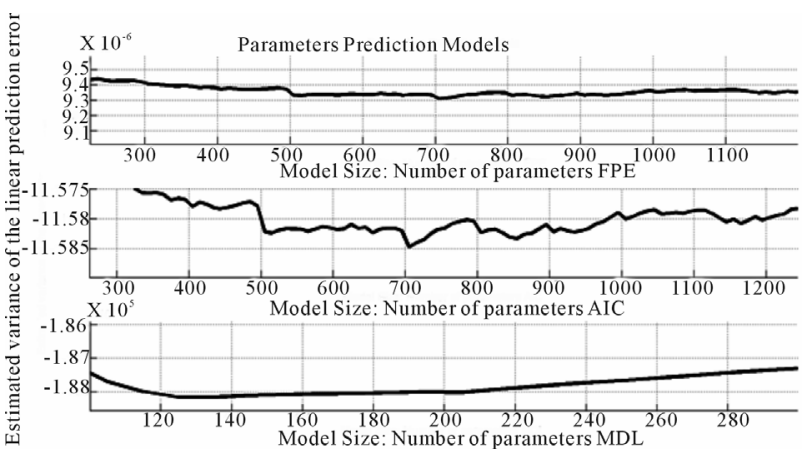

Figure 5. Parameter prediction methods, using Table 1 we calculate the number of parameters, for a signal with four frequency components: $1 \mathrm{MHz}, 1.01 \mathrm{MHz}, 1.05 \mathrm{MHz}$ and $1.1 \mathrm{MHz}$

guish the minimum $\sigma_{w p}^{2}$, which for the FPE and AIC techniques is around 700 parameters, while with the MDL technique $\sigma_{w p}^{2}$ is minimized with 130 parameters.

Using both suggestions, we obtain the spectra shown in Figure 6.

It is important to note two facts in this exercise.

1) The frequency resolution criterion, and not the tra- 
ditional "trial and error" method, is considered when evaluating the number of parameters. The acquired signal fully meets the Nyquist criterion, but the frequency resolution had to be considered also to obtain a reliable interval of the number of parameters using the equations in Table 1.

2) The frequency resolution allowed certainty in the techniques used to evaluate the number of parameters and gives a wide interval of parameters that give a good result. This is reflected in Figure 5, which shows a minimum value for all methods and in Figure $\mathbf{6}$ where the four peaks are identified.

\section{Frequency Resolution: In Turbulent Signals from a Supersonic Jet}

The previous exercise, in which we used deterministic signals, showed that if frequency resolution is taken into account, the results obtained from the parameter evaluation techniques give more precise results. In the following exercise, we will demonstrate that this result is also valid for random signals. The signals presented below come from the laser light scattered by a high speed jet.

The signal that comes out of the photo detector is proportional to spatial Fourier transform as a function of time, of the density fluctuation for a wave vector determined by the optical set-up. The signal should contain then the entropic and the acoustic modes described in Section 2. Figure 7 shows the spectral density of such a signal obtained with a spectrum analyzer. The acoustic peak should appear around $2 \mathrm{MHz}$. The frequency of the entropy peak depends on the local speed and on the direction of the wave vector. When the latter is in the direction of the flow, the frequency corresponds to the Doppler frequency and is higher than the acoustic. If the wave vector is perpendicular to the flow, the average frequency is around zero. Previous papers [5-11], have reported an unexpected third peak, which can be identified as Mach-3C in Figure 2, and whose origin is still

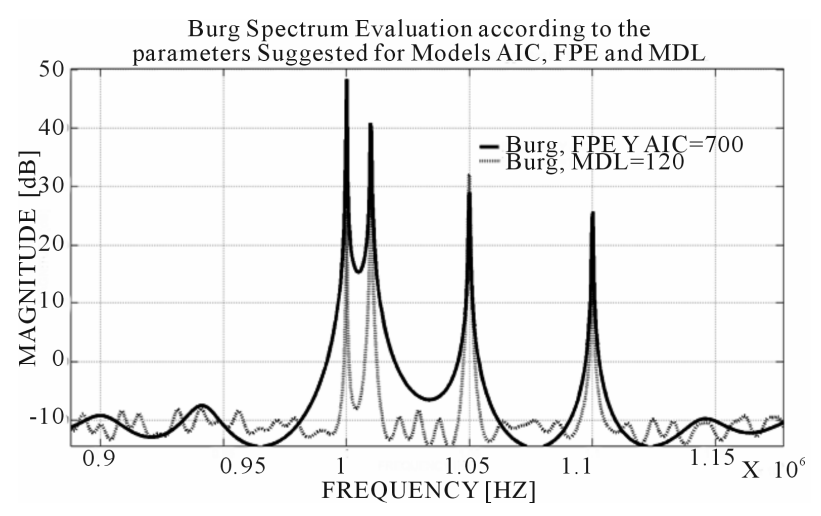

Figure 6. Spectrum of a signal with four components, obtained with the Burg parametric periodogram, using the parameters suggested by the prediction methods.

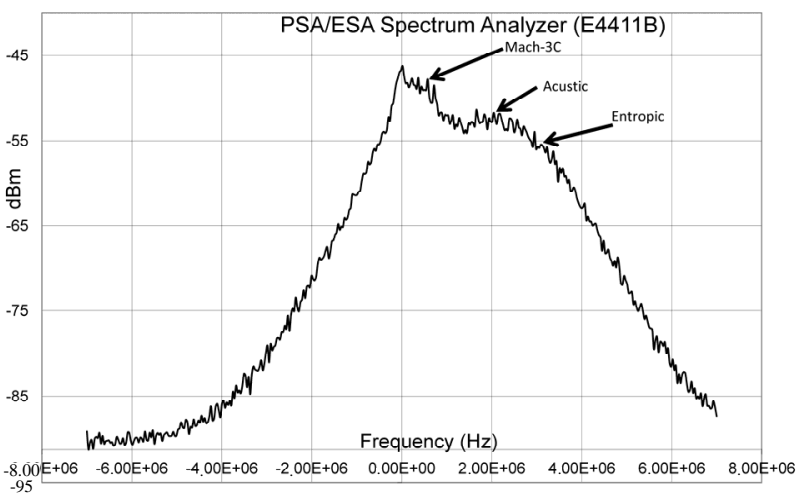

Figure 7. Spectral density displayed in a spectrum analyzer.

subject of investigation.

In Figure 7, the peaks mentioned are not easily identified by the untrained eye. The signal was acquired with an oscilloscope Agilent Infinitum Model 54830B. The sampling frequency was $f_{s}=40 \mathrm{MHz}$ and the number of samples was 65,553 samples, giving a frequency resolution $\Delta f=610 \mathrm{~Hz}$, and then fed to the spectrum analyzer. The acquisition parameters were automatically determined by the instrument, without possibility of any control from the user.

The techniques presented in Table $\mathbf{1}$ were applied to the signal; the evaluation of the number of parameters needed for a Burg parametric periodogram is shown in Figure 8. The Burg periodogram was chosen because it has good resolution for low-amplitude and low energy components [10,11].

In Figure 8 one can observe that for the AIC and FPE models, there is no tendency to minimize the estimated variance of the linear prediction error, while the MDL model predicts that the variance should show a minimum for around 160 parameters.

Figure 9 shows the various spectral graphs of the above mentioned time signal obtained with the Burg periodogram with different number of parameters considering those predicted by the techniques. The interval of parameter variation is between 90 and 300. The number suggested by the MDL is within the range. Figure 9 shows peaks that appear and disappear when the number of parameters changes, without any particular tendency in relation to the behavior of the peaks.

The peak that we are interested in should appear at around $2 \mathrm{MHz}$, so following Nyquist's Theorem, the signal was acquired properly. It could be suggested that filters and other processing tools (decimation, windows, etc.) might improve the acquired signal in order to obtain more precise frequency information. However, once the signal has been acquired, we cannot observe more than what the frequency resolution allows. Therefore, the desired frequency resolution has to be taken into account before the acquisition of the signal $[10,11]$. 


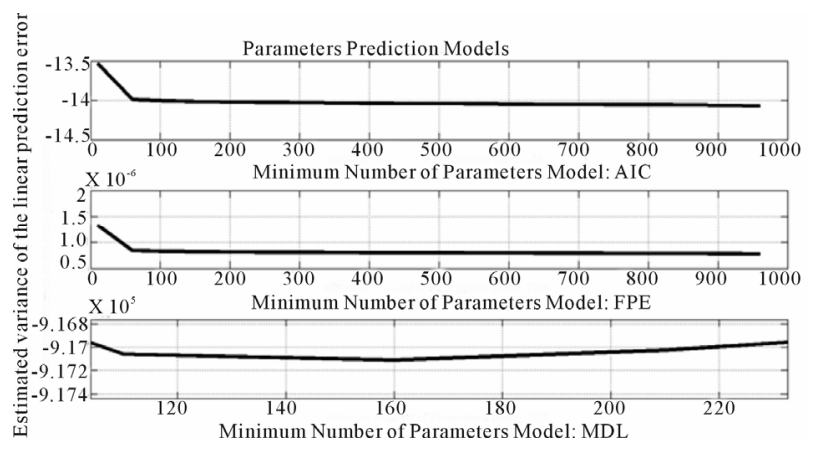

Figure 8. Using Table 1 we calculate the number of parameters, for the evaluation of the signal whose spectrum is shown in Figure 7.

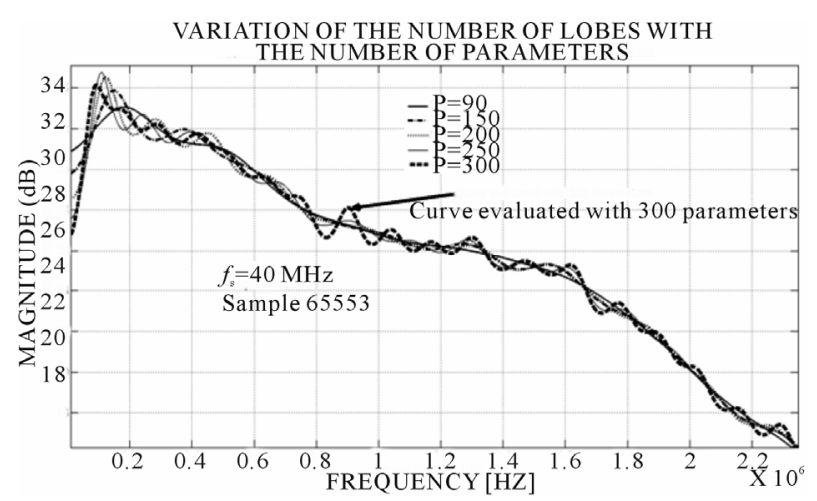

Figure 9. Burg periodograms evaluated with different parameters; using the minimum values of the graphs presented in Figure 8. Modeling the spectrum of Figure 7.

In exactly the same experimental conditions, a new signal was acquired considering, this time, a frequency resolution of $\Delta f=122070 \mathrm{~Hz}$. The evaluation of the number of parameters in this case is shown in Figure 10. It is interesting to note that the three parameters prediction methods show a minimum in the estimated variance of the linear prediction error. Two of these predict 150 parameters.

Figure 11 shows the corresponding spectrum calculated with a Burg periodogram using 150 parameters. The three expected peaks may clearly be observed [11].

This result clearly shows that there must be a close relationship between the frequency resolution of the acquired signal and the number of parameters. Therefore, the parameter prediction methods should explicitly consider the frequency resolution.

As a final proof of the importance of the frequency resolution, Figure 12 shows the spectrum of the signal evaluated with different number of parameters. In this Figure 12, the three lobes appear in a wide interval of parameters. The fact that three modes of density fluctuations inside the flow can be resolved is a new and important result. Two modes were expected and the origin of the third one is under study.
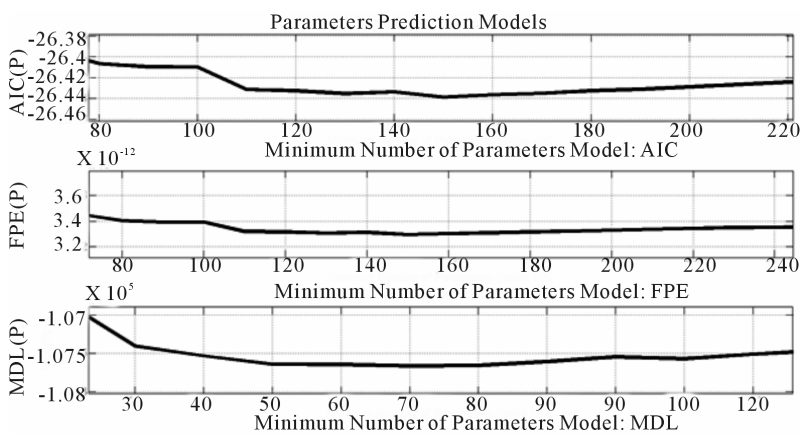

Figure 10. Evaluation of the number of parameters for a turbulent signal with $\Delta f=122070 \mathrm{~Hz}$. Using Table 1 , we calculate the number of parameters.

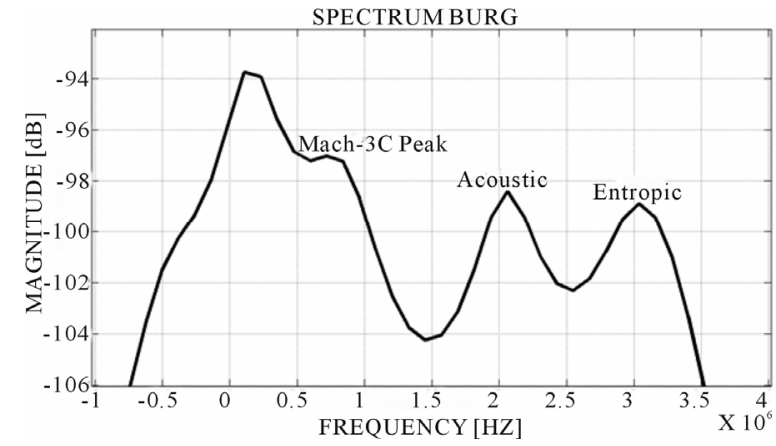

Figure 11. Spectrum evaluated considering the criteria referred to in this paper. Using the minimum values of the graphs presented in Figure 10.

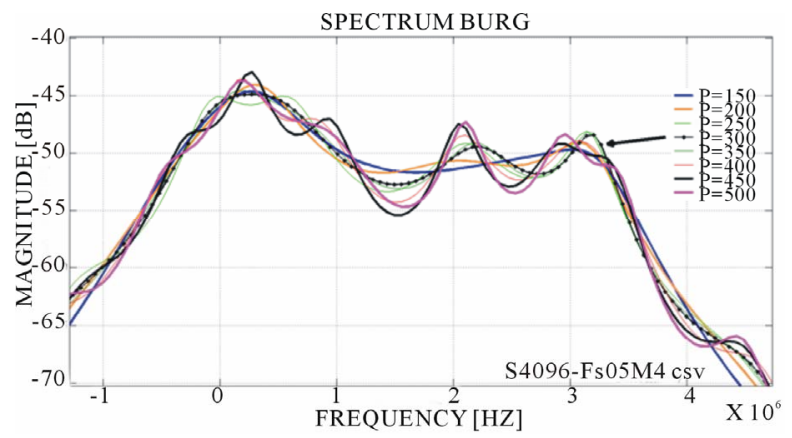

Figure 12. Spectra of a same signal, $\Delta f=122070 \mathrm{~Hz}$, with a different number of parameters.

Figures 11 and 12 prove the importance of taking into account the frequency resolution before acquiring a signal; Nyquist's theorem alone is not enough. As it may be seen in Figure 12, when a good acquisition is made, the shape of the spectrum does not vary over a wide parameter interval, thus ensuring the shape of the spectrum. This is valid in turbulent as well as in deterministic signals.

\section{Summary and Conclusions}

We presented two different kinds of signals for which we could determine the optimal number of parameters through 
the minimum in the graph $\sigma_{w p}^{2}$ vs $p$. To do so, we had to choose the frequency resolution $\Delta f$. This choice is simple when the signals are well known as in the first example. The four frequencies of the original signal were clearly identified. For the second example, where the signal is random, the resolution depends on previous knowledge of the experiment. In our case, entropic and acoustic peaks expected from the literature, were resolved. Furthermore, a third low frequency non expected peak, was studied with respect to the position in the jet where the signal came from.

This paper gives a more objective way to determine the number of parameters and a better spectral density. It is surprising that even though it is well known that high temporal resolution implies low frequency resolution, when sampling a signal, the Nyquist theorem is applied blindly without taking into account the final use of the data. This is an important result in signal processing that can be seen by comparing Figure $\mathbf{7}$ with Figure 11.

A Rayleigh scattering technique combined with the heterodyne detection of light scattered by the molecules of a transparent gas was used to detect density fluctuations. The periodograms helped resolve various frequentcies and gave more insight on the internal structure of the jet.

The periodograms are parametric signal processing tools that allow the modeling of a signal. To properly implement them, it is necessary to determine the optimal number of parameters, which ensures that the signal has been modeled correctly. The theory predicts that many parameters could add spurious peaks, and that few parameters may not reproduce the signal properly. The results presented in this paper show that the resolution $\Delta f$ could be an important factor in finding the optimal number of parameters required to model a signal by using parametric periodograms.

In the future, we expect to determine clearly how to obtain the optimal number of parameters directly from the frequency resolution.

\section{Acknowledgements}

To the PAPIIT IN117712 project "Propagación de ondas a través de interfaces”.

\section{REFERENCES}

[1] B.-T. Chu and L. S. G. Kovásznay, "Non-Linear Interactions in a Viscous Heat-Conducting Compressible Gas," Journal of Fluid Mechanics, Vol. 3, No. 5, 1958, pp. 494514. http://dx.doi.org/10.1017/S0022112058000148

[2] Y. Andreopoulos, J. Agui and G. Brassulis, "Shock Wave -Turbulence Interactions," Annual Review of Fluid Mechanics, Vol. 32, 2000, pp. 309-345. http://dx.doi.org/10.1146/annurev.fluid.32.1.309

[3] D. Bogdanoff, "Compressibility Effects in Turbulet Shear Layer,” AAIA Journal, Vol. 21, No. 6, 1983, pp. 926-927. http://dx.doi.org/10.2514/3.60135

[4] C. E. Stern, "Diffusion Rayleigh et Detection Heterodyne Optique. Application Aux Fluctuations Atmospheriques,” Ph.D. Dissertation, L’Univeersite De Paris Sud Centre D’Osay, Paris, 1981.

[5] C. Aguilar, "Diseño y Construcción de un Sistema de Difusión Rayleigh para Estudiar Fluctuaciones de Densidad en Gases Transparentes,” Thesis Dissertations, Facultad de Ciencias UNAM, México D.F., 2003.

[6] C. Azpeitia, "Estudio Experimental no Instrusivo de las Fluctuaciones de Densidad Dentro de un jet Turbulento," Thesis Dissertations, Facultad de Ciencias UNAM, México D.F, 2004.

[7] C. E. Stern, F. J. M. Alvarado and C. Aguilar, "Density Measurements in a Supersonic Jet,” Journal of Mechanics of Materials and Structures, Vol. 2, No. 8, 2007, pp. 1437-1448. http://dx.doi.org/10.2140/jomms.2007.2.1437

[8] C. E. Stern, C. Aguilar and J. M. Alvarado, "Density Fluctuations in a Supersonic Jet," 12th AIAA/CEAS Aeroacoustics Conference, Cambridge Massachusetts, EEUU, May 2006, pp. 1-7.

[9] C. Aguilar, C. Azpetia, J. M. Alvarado and C. E. Stern, "Study of Mach Line and Acoustic Waves in a Jet Using Rayleigh Scattering,” 43th Aerospace Sciences Meeting (AIAA), Reno, EEUU, 2005.

[10] J. M. Alvarado, “Analisis Espectral de Señales Obtenidas a Partir de Disfusion Rayleigh,” M.E. Thesis Dissertations, Facultad de Ingenieria UNAM, Mexico D.F., 2004.

[11] J. M. Alvarado, "Técnicas Modernas para el Tratamiento de SeñalesTurbulentas,” Ph.D. Dissertations, Facultad de Ingenieria UNAM, Mexico D.F., 2010.

[12] S. Kay, "Modern Spectral Estimation,” Theory \& Application, 4th Edition, Signal Processing Series, Englewood Cliffs, Prentice Hall, 1988.

[13] S. Kay and S. Lawrence, "Model Order Selection-Parte II, Spectrum Analysis-An Modern Perspective,” IEEE Proceedings, Vol. 69, No. 11, 1981, pp. 1395-1398.

[14] J. Proakis, G. Manolakis and G. Dimintris, "Selection of AR Model Order,” Digital Signal Processing. Principles, Algorithms and Aplications, 3th Edition, Prentice Hall, Upper Saddle River, 1998, p. 932.

[15] C. Therrien, “Order Selecction,” Discrete Random Signal and Statistical Signal Processing, Englewood Cliffs, Prentice Hall, 1992, pp. 542-560.

[16] E. Ifeachor and B. Jervis, "The Periodogram Method and Periodogram Properties,” Digital Signal Processing: A Practical Approach, 2nd Edition, Edinburgo Gate, Prentice Hall, 2002, pp. 703-719.

[17] J. M. Alvarado and C. E. Stern, "Un Complemento al Teorema de Nyquist,” Revista Mexicana de Física, Vol. 56, No. 2, 2010, pp. 165-171. 